\title{
The Transfer Functions of Rocket Nozzles
}

\author{
H. S. TSIEN ${ }^{1}$
}

Daniel and Florence Guggenheim Jet Propulsion Center, California Institute of Technology, Pasadena, Calif.

\begin{abstract}
The transfer function is defined as the fractional oscillating mass flow rate divided by the fractional sinusoidal pressure oscillation in the rocket combustion chamber. This is calculated as a function of the frequency of oscillation. For very small frequencies, the transfer function is approximately 1 with a small "lead component." For very large frequencies, the transfer function is considerably larger than 1 , and is approximately $1+\left(\gamma M_{1}\right)^{-1}$ where $\gamma$ is the ratio of specific heats of the gas, and $M_{1}$ is the Mach number at entrance to the nozzle.
\end{abstract}

$\mathbf{R}$ ECENTLY, the problem of combustion instability of rocket motor has been studied by several authors $(1,2$, and 3$) .^{2}$ In these investigations, it is assumed that the percentage increase of the mass rate of flow through the nozzle is equal to the percentage increase of pressure in the rocket cylinder. It is, however, not certain whether this assumption is correct. Since this flow condition enters in a direct manner into the instability calculation, the relation between flow variations and the pressure variations should be determined more carefully. It is the purpose of this paper to do this. The result of the present study is expressed as the transfer function of the rocket nozzle, i.e., the ratio of the fractional increases in mass flow and the chamber pressure as function of the frequency of the oscillation. It indicates that the transfer function is rather a complex function of the nozzle geometry and the frequency of oscillation, and that the previous very simple assumption is only justified for very small frequencies of oscillation.

\section{Flow Conditions}

The flow in the nozzle will be considered as onedimensional, i.e., at each nozzle section the conditions are taken to be uniform and the independent variables of the problem are then the time $t$ and the distance $x$ along the nozzle axis in the direction of flow (Fig. 1). Let $p$ be the pressure, $\rho$ the density, and $u$ the velocity. The primed quantities are the oscillating quantities; thus $\rho^{\prime}$ is the density oscillation. Similarly, the unprimed quantities are the steady-state or undisturbed quantities. Therefore $p^{\prime} / p$ is the oscillating pressure at $x$ expressed as fraction of the steady-state pressure at $x$. These fractional quantities are assumed to be small so that only first order terms need to be considered. Since the mass rate of flow per unit area is

Received January 2, 1952.

1 Robert H. Goddard Professor of Jet Propulsion.

${ }^{2}$ Numbers in parentheses refer to References on page 162.
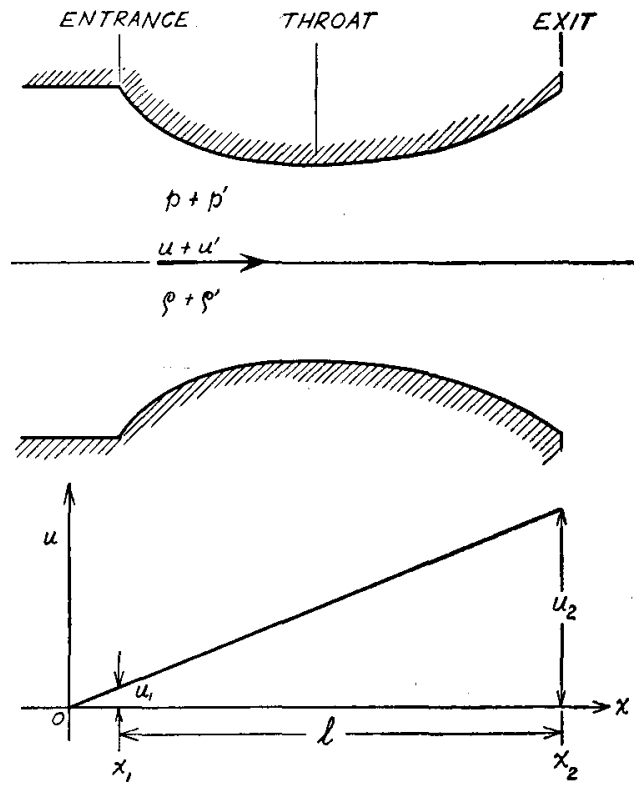

FIG. 1 ROCKET NOZZLE WITH LINEAR VELOCITY DISTRIBUTION

equal to the product of density and velocity, the fractional increment of the mass rate of flow is $\left(\frac{\rho^{\prime}}{\rho}+\frac{u^{\prime}}{u}\right)$. Hence the purpose of this paper can now be stated as simply to compute the ratio $\left(\frac{\rho^{\prime}}{\rho}+\frac{u^{\prime}}{u}\right) /\left(\frac{p^{\prime}}{p}\right)$ at the entrance to the nozzle as a function of the oscillation frequency $\omega$. This ratio will be called the transfer function of the rocket nozzle.

The conditions at the entrance to the nozzle are fixed by the plausible assumption that the temperature of the combustion gas is not changed by variations in pressure. Let the gas be considered as a perfect gas, and let the subscript 1 denote the entrance to the nozzle; then

$$
\left(\frac{p^{\prime}}{p}\right)_{1}-\left(\frac{\rho^{\prime}}{\rho}\right)_{1}=0 \ldots \ldots \ldots \ldots \ldots \ldots[1]
$$

Since the flow in the divergent part of the rocket nozzle is supersonic, the obvious additional condition for the complete determination of the flow is simply that the propagation of oscillations must be toward the downstream direction. For instance, if $U(x)$ is the velocity of the propagation of density oscillations at the section $x$, then for an observer who travels with the speed $U$, the density is stationary, i.e.,

$$
\frac{\partial}{\partial t}\left(\frac{\rho^{\prime}}{\rho}\right)+U \frac{\partial}{\partial x}\left(\frac{\rho^{\prime}}{\rho}\right)=0
$$

This is the equation for determining the velocity of 
propagation. The condition of downstream propagation means positive $U$. Thus, in the nozzle the signs of $\partial\left(\frac{\rho^{\prime}}{\rho}\right) / \partial t$ and $\partial\left(\frac{\rho^{\prime}}{\rho}\right) / \partial x$ must be opposite. Sïmilarly, the signs of $\partial\left(\frac{u^{\prime}}{u}\right) / \partial t$ and $\partial\left(\frac{u^{\prime}}{u}\right) / \partial x$ must also be opposite in the nozzle.

\section{Formulation of the Problem in Nozzle}

In the nozzle, if $A$ is the cross section of the nozzle at $x$, then the continuity equation is

$$
A \frac{\partial \rho^{\prime}}{\partial t}+\frac{\partial}{\partial x}\left[A\left(\rho+\rho^{\prime}\right)\left(u+u^{\prime}\right)\right]=0 .
$$

The steady-state flow or the undisturbed flow satisfies, however, the simpler continuity equation

$$
A \rho u=\text { const }
$$

By eliminating $A$ from Equation [3] by this equation, and by retaining only the first-order terms of the oscillating quantities, one obtains the linearized continuity equation as

$$
\frac{\partial}{\partial t}\left(\frac{\rho^{\prime}}{\rho}\right)+u \frac{\partial}{\partial x}\left(\frac{\rho^{\prime}}{\rho}+\frac{u^{\prime}}{u}\right)=0
$$

Similarly, the dynamic equation is

$$
\begin{aligned}
\frac{\partial}{\partial t}\left(\frac{u^{\prime}}{u}\right)+\left(\frac{\rho^{\prime}}{\rho}+2 \frac{u^{\prime}}{u}\right) \frac{d u}{d x}+u & \frac{\partial}{\partial x}\left(\frac{u^{\prime}}{u}\right)= \\
& \left(\frac{p^{\prime}}{p}\right) \frac{d u}{d x}-\frac{p}{\rho u} \frac{\partial}{\partial x}\left(\frac{p^{\prime}}{p}\right) .
\end{aligned}
$$

For any fluid mass, the cntropy is maintained. Therefore, if $\gamma$ is the ratio of specific heats of the gas,

$$
\left(\frac{\partial}{\partial t}+u \frac{\partial}{\partial x}\right)\left[\left(\frac{p^{\prime}}{p}\right)-\gamma\left(\frac{\rho^{\prime}}{\rho}\right)\right]=0
$$

Equations [4], [5], and [6] are three equations for the three dependent variables $\left(\rho^{\prime} / \rho\right),\left(p^{\prime} / p\right)$, and $\left(u^{\prime} / u\right)$.

It is convenient in the following calculations to introduce a specific nozzle shape such that the steady velocity in the nozzle increase simply linearly with $x$ (Fig. 1). The simplest way to specify this is

$$
u=\frac{u_{1}}{x_{1}} x .
$$

where the subscript 1 denotes again quantities at the entrance to the nozzle. $\quad x_{1}$ is thus the $x$-coordinate at the entrance to the nozzle. Therefore, the origin of the $x$-axis is not generally at the entrance to the nozzle; it is there only if the steady velocity at entrance is equal to zero. With Equation [7], Equation [6] becomes

$$
\left(\frac{\partial}{\partial t}+\frac{u_{1}}{x_{1}} \frac{\partial}{\partial \log x}\right)\left[\left(\frac{p^{\prime}}{p}\right)-\gamma\left(\frac{\rho^{\prime}}{\rho}\right)\right]=0 .
$$

Therefore, if the entropy oscillations at the entrance to the nozzle is $\epsilon e^{i \omega t}$, i.e.,

$$
\left(\frac{p^{\prime}}{p}\right)_{1}-\gamma\left(\frac{\rho^{\prime}}{\rho}\right)_{1}=\epsilon e^{i \omega t} \ldots \ldots \ldots \ldots[9]
$$

then, according to Equation [8] in general

$$
\left(\frac{p^{\prime}}{p}\right)-\gamma\left(\frac{\rho^{\prime}}{\rho}\right)=\epsilon \exp \left[i \omega\left(t-\frac{x_{1}}{u_{1}} \log \frac{x}{x_{1}}\right)\right] \ldots
$$

By eliminating $\left(p^{\prime} / p\right)$ between Equations [5] and [10], the resultant equation together with Equation [4] constitute a system of two equations for the two unknowns $\left(\rho^{\prime} / \rho\right)$ and $\left(u^{\prime} / u\right)$.

Now let $x_{2}$ be the $x$-coordinate at the exit of the nozzle, or if $l$ is the length of the nozzle,

$$
x_{2}=x_{1}+\ell \text {. }
$$

Define a nondimensional independent variable $\xi$ as

$$
\xi=x / x_{2} \ldots \ldots \ldots \ldots \ldots \ldots
$$

Then

$$
\xi_{1}=x_{1} / x_{2}=x_{1} /\left(x_{1}+l\right)=\mathbf{1} /\left(\mathbf{1}+\frac{l}{x_{1}}\right)
$$

The oscillating quantities with frequency $\omega$ can now be written as

$$
\begin{aligned}
& \left(\frac{\rho^{\prime}}{\rho}\right)=f(\xi) e^{i \omega t} \\
& \left(\frac{u^{\prime}}{u}\right)=g(\xi) e^{i \omega t}
\end{aligned}
$$

By introducing these expressions into the differential equations for $\rho^{\prime} / \rho$ and $u^{\prime} / u$, equations for $f$ and $g$ are derived. The results can be, however, written more compactly if one uses the reduced frequency $\beta$, defined as

$$
\beta=\frac{\omega x_{1}}{u_{1}}=\frac{\omega x_{2}}{u_{2}}=\frac{\omega l}{u_{2}} \frac{1}{1-\xi_{1}}
$$

where $u_{2}$ is the exit velocity of the nozzle. $\beta$ is then the ratio of the angular frequency $\omega$ and the velocity gradient $u_{1} / x_{1}$ along the nozzle axis. Then from Equations [4], [5], and [10] the equations for the $f$ and $g$ are obtained as

$$
\xi\left[\frac{d f}{d \xi}+\frac{d g}{d \xi}\right]+i \beta f=0
$$

and

$$
\begin{aligned}
(2+i \beta) g+( & 1-i \beta) f-\xi \frac{d f}{d \xi}= \\
& \gamma f-\frac{1}{M^{2}} \xi \frac{d f}{d \xi}+\epsilon\left(\frac{\xi}{\xi^{\mathrm{I}}}\right){ }^{i \beta}\left[1+\frac{i \beta}{\gamma M^{2}}\right] .
\end{aligned}
$$

where $M$ is the local Mach number of the undisturbed flow at $x$ or $\xi$. In fact, for the assumed linear velocity distribution in the nozzle,

$$
\begin{gathered}
M^{2}=\frac{M_{1}{ }^{2} \xi^{2}}{\xi_{1}{ }^{2}-\frac{\gamma-1}{2} M_{1}{ }^{2}\left(\xi^{2}-\xi_{1}^{2}\right)}= \\
\cdot \frac{M_{1}{ }^{2}\left(\frac{x}{x_{1}}\right)^{2}}{1-\frac{\gamma-1}{2} M_{1}{ }^{2}\left(\frac{x^{2}}{x_{1}{ }^{2}}-1\right)} \ldots \ldots
\end{gathered}
$$

$M_{1}$ is the Mach number at the entrance to the nozzle.

By eliminating $g(\xi)$ from Equations [17] and [18], a single second-order equation for $f(\xi)$ is obtained. The result can, however, be reduced to more convenient form by using a new independent variable $z$ defined as

$$
z=\frac{\frac{\gamma+1}{2} M_{1}{ }^{2}\left(\xi / \xi_{1}\right)^{2}}{1+\frac{\gamma-1}{2} M_{1}{ }^{2}}
$$


It is easy to show that $z$ is actually the square of the ratio of $u$ to the so-called critical sound speed. Thus $z=1$ at the throat of the nozzle. In terms of $z$, the differential equation for $f$ is

$$
\begin{aligned}
& z(1-z) \frac{d^{2} f}{d z^{2}}-\left\{2+\frac{2 i \beta}{(\gamma+1)}\right\} z \frac{d f}{d z}-\frac{i \beta(2+i \beta)}{2(\gamma+1)} f= \\
& -i \beta \epsilon\left(\frac{z}{z_{1}}\right)^{-i(\beta / 2)}\left[\frac{1-i \beta \frac{\gamma-1}{2 \gamma}}{2(\gamma+1)}+\frac{2+i \beta}{4 \gamma} \frac{1}{z}\right] \ldots \ldots
\end{aligned}
$$

The relation between $f(z)$ and $g(z)$ is

$$
\begin{aligned}
& (2+i \beta) g(z)=[(\gamma-1)+i \beta] f(z)-(\gamma+1)(1-z) \frac{d f}{d z}+ \\
& \epsilon\left(\frac{z}{z_{1}}\right)^{-i(\beta / 2)}\left[1-\frac{i \beta(\gamma-1)}{2 \gamma}+\frac{i \beta(\gamma+1)}{2 \gamma} \frac{1}{z}\right] \ldots \ldots
\end{aligned}
$$

$z_{1}$ is, of course, the value of $z$ corresponding to $\xi_{1}$ as given by Equation [13]; i.e.,

$$
z_{1}=\frac{\frac{\gamma+1}{2} M_{1}^{2}}{1+\frac{\gamma-1}{2} M_{1}^{2}}
$$

The initial condition needed to solve Equation [21] is specified by Equations [1] and [9]. That is, at $x=x_{1}$ or $z=z_{1}$,

$$
f\left(z_{1}\right)=-\frac{\epsilon}{\gamma-1}
$$

The complete solution requires the cognizance of the fact that the propagation of oscillations must be toward the downstream direction as discussed in the previous section.

When $f(z)$ is determined, one can compute $g(z)$ by Equation [22]. Then the density oscillations and the velocity oscillations are determined as functions proportional to the amplitude of entropy oscillation $\epsilon$. Since the point of interest is the ratio of the oscillations, the arbitrary $\epsilon$ does not really enter into the final result. In fact, according to Equation [1], the ratio of the fractional increase in mass rate of flow and that of pressure, or the transfer function $G(\beta)$ is

$$
\begin{aligned}
& G(\beta)=\left[\left(\frac{\rho^{\prime}}{\rho}\right)_{1}+\left(\frac{u^{\prime}}{u}\right)_{1}\right] /\left(\frac{p^{\prime}}{p}\right)_{1}= \\
& {\left[\left(\frac{\rho^{\prime}}{\rho}\right)_{1}+\left(\frac{u^{\prime}}{u}\right)_{1}\right]\left(\frac{\rho^{\prime}}{\rho}\right)_{1}=1+\frac{g\left(z_{1}\right)}{f\left(z_{1}\right)} \ldots \ldots}
\end{aligned}
$$

Therefore $G(\beta)$ is unity as assumed by previous investigators of combustion instability only if there is no velocity oscillation at the entrance, or $\left(u^{\prime} / u\right)_{1}=0$. It is perhaps worthwhile to point out the fact that the present definition of transfer function $G(\beta)$ corresponds to the practice of servomechanism analysis, if the pressure oscillation $\left(p^{\prime} / p\right)_{1}$ is taken as the "input" and the mass flow oscillation as the "output." Hence the transfer function $G(\beta)$ will be useful also in the synthesis of the systems for servocontrol or servostabilization of the rocket motor, as suggested by W. Bollay (4).

\section{Solution for Small Frequency}

The basic differential equation with linear velocity distribution in the nozzle as given by Equation [21] can be reduced to the hypergeometric differential equation by the substitution $f(z)=z \cdot w(z) . \quad w(z)$ is then the hypergeometric function. However, the calculation is rather difficult. For a first discussion, only cases of either very small frequency or very large frequency will be considered here.

If the frequency $\beta$ is very small, the functions $f(z)$ and $g(z)$ can be expanded in terms of this parameter

$$
\begin{aligned}
& f(z ; \beta)=f^{(0)}(z)+\beta f^{(1)}(z)+\ldots \\
& g(z ; \beta)=g^{(0)}(z)+\beta g^{(1)}(z)+\ldots
\end{aligned}
$$

These expressions are appropriate if the interval of $z$ concerned does not include $z=0$. Fortunately, the physical problem generally specifies nonvanishing $u$ at the entrance to the nozzle. Therefore, $\xi_{1} \neq 0$ and thus $z_{1} \neq 0$. The restriction on the validity of the expansions is thus generally complied. By substituting Equations [26] and [27] into the basic Equations [21] and [22], and then equating terms of same powers in $\beta$, one has

$$
z(1-z) \frac{d^{2} f^{(0)}}{d z^{2}}-2 z \frac{d f^{(0)}}{d z}=0
$$

and

$$
\begin{aligned}
z(1-z) \frac{d^{2} f^{(1)}}{d z^{2}}-2 z \frac{d f^{(1)}}{d z}=i\left\{\frac{2}{\gamma+1} z \frac{d f^{(0)}}{d z}+\frac{1}{\gamma+1} f^{(0)}-\right. \\
\left.\frac{\epsilon}{2}\left(\frac{1}{\gamma+1}+\frac{1}{\gamma z}\right)\right\} \ldots \ldots[29]
\end{aligned}
$$

Furthermore

$$
\begin{aligned}
2 g^{(0)}(z)= & (\gamma-1) f^{(0)}(z)-(\gamma+1)(1-z) \frac{d f^{(0)}}{d z}+\epsilon \ldots[30] \\
2 g^{(1)}(z)= & (\gamma-1) f^{(1)}(z)-(\gamma+1)(1-z) \frac{d f^{(1)}}{d z}+i\left[f^{(0)}(z)-\right. \\
& \left.g^{(0)}(z)\right]-\frac{i \epsilon}{2}\left[\log \left(\frac{z}{z_{1}}\right)+\frac{\gamma-1}{\gamma}-\frac{\gamma+1}{\gamma z}\right] \ldots \ldots[31]
\end{aligned}
$$

The initial condition of Equation [24] now becomes

$$
\begin{array}{r}
f^{(0)}\left(z_{1}\right)=-\frac{\epsilon}{\gamma-1} \\
f^{(1)}\left(z_{1}\right)=0 \ldots
\end{array}
$$

A straightforward calculation will give the following relation for the propagation velocity $U$ for the density fluctuation defined by Equation [2],

$$
\frac{U}{u}=-\frac{i \beta f(z)}{2 z \frac{d f}{d z}}=-\frac{1}{2 z} \frac{i \beta\left[f^{(0)}(z)+\beta f^{(1)}(z) \ldots \ldots\right]}{\frac{d f^{(0)}}{d z}+\beta \frac{d f^{(1)}}{d z}+\ldots \ldots}
$$

where $u$ is, of course, the undisturbed flow velocity at the section concerned. Therefore, in order that the propagation speed be finite even for small $\beta, f^{(0)}(z)$ must be a constant. Hence the appropriate solution of Equation [28] together with the condition of Equation $[32]$ is

$$
f^{(0)}(z)=-\frac{\epsilon}{\gamma-1}
$$

Then Equation [30] gives immediately

$$
g^{(0)}(z)=0 \text {. }
$$

Up to this order of approximation then, $G=1$, as shown by Equation [25]. Therefore the assumption of the previous investigators of the combustion in- 
stability of rocket motor is indeed correct if the frequency is vanishingly small.

With $f^{(0)}(z)$ given by Equation [35], the equation for $f^{(1)}(z)$, Equation [29], can be written as

$$
\frac{d}{d z}\left[(1-z)^{2} \frac{d f^{(1)}}{d z}\right]=-\frac{i \epsilon}{2}\left[\frac{1}{\gamma-1}+\frac{1}{\gamma z}\right] \frac{1-z}{z}
$$

Therefore the solution is

$$
\begin{aligned}
& d f^{(1)}=-{ }_{2} \epsilon {\left[\frac{1}{\gamma(\gamma-1)} \frac{\log z}{(1-z)^{2}}+\frac{1}{(\gamma-1)} \frac{1}{(1-z)}-\right.} \\
&\left.\frac{1}{\gamma} \frac{1}{z(1-z)}\right]+\frac{C}{(1-z)^{2}}
\end{aligned}
$$

The quantity inside the square bracket is finite and positive for $z>0$, while the last term is infinite at the throat of the nozzle where $z=1$. Hence, according to Equation [34], the condition for finite positive propagation speed $U$ requires $C=0$. Therefore ${ }^{3}$

$$
\begin{array}{r}
d f^{(1)}={ }_{2}^{i \epsilon}\left[-\frac{1}{\gamma(\gamma-1)} \frac{\log z}{(1-z)^{2}}-\frac{1}{(\gamma-1)} \frac{1}{(1-z)}+\right. \\
\left.\frac{1}{\gamma} \frac{1}{z(1-z)}\right] \ldots
\end{array}
$$

This equation together with Equations [31] and [33] then gives

$$
g^{(1)}\left(z_{1}\right)=\frac{i \epsilon}{4 \gamma}\left\{\frac{\gamma+1}{\gamma-1} \frac{\log z_{1}}{1-z_{1}}+1\right\}
$$

Therefore, up to the first order in $\beta$, the transfer function $G(\beta)$ is

$$
\begin{aligned}
& G(\beta) \cong 1+\frac{\beta g^{(1)}\left(z_{1}\right)}{f\left(z_{1}\right)}=1+i \beta\left\{\frac{\gamma+1}{4 \gamma} \frac{\log \left(\frac{1}{z_{1}}\right)}{1-z_{1}}-\right. \\
&\left.\frac{\gamma-1}{4 \gamma}\right\}, \quad \beta \ll 1 \ldots \ldots[39]
\end{aligned}
$$

Therefore, when the frequency $\beta$ is not exactly zero, the transfer function has a small "lead component," proportional to the frequency.

\section{Solution for Large Frequencies}

If the value of $\beta$ is very large, one must find the asymptotic solution of Equation [21]. The dominating terms in Equation [21] are

$$
\begin{aligned}
& z(1-z) \frac{d^{2} f}{d z^{2}}-\frac{2 i \beta}{\gamma+1} z \frac{d f}{d z}+\frac{\beta^{2}}{2(\gamma+1)} f= \\
& \beta^{2} \epsilon\left(\frac{z}{z_{1}}\right)^{-(i \beta / 2)} \frac{1}{4 \gamma}\left[\frac{1}{z}-\frac{\gamma-1}{\gamma+1}\right] .
\end{aligned}
$$

For the particular integral $f^{*}$, take

$$
f^{*}(z)=Z(z)\left(\frac{z}{z_{1}}\right)^{-(i \beta / 2)}
$$

w'iere $Z(z)$ is a function of $z$ not involving $\beta$. Therefore, by retaining only the highest order term,

$$
\begin{aligned}
\frac{d f^{*}}{d z} \cong-\frac{i \beta}{2} \frac{Z(z)}{z}\left(\frac{z}{z_{1}}\right)^{-(i \beta / 2)} \\
\frac{d^{2} f^{*}}{d z^{2}} \cong-\frac{\beta^{2}}{4} \frac{Z(z)}{z^{2}}\left(\frac{z}{z_{1}}\right)^{-(i \beta / 2)}
\end{aligned}
$$

\footnotetext{
${ }^{3}$ Professor L. Crocco has very kindly pointed out that the condition for positive finite propagation speed $U$ is equivalent to the suppression of all singularities at the sonic point. This observation may simplify the analysis for general values of $\beta$.
}

By substituting these derivatives into Equation [40], it is found that

$$
Z(z)=-\frac{\epsilon}{\gamma}
$$

thus

$$
f^{*}(z)=-\frac{\epsilon}{\gamma}\left(\frac{z}{z_{1}}\right)^{-(i \beta / 2)}
$$

To find the complementary function, let

$$
f(z)=e^{i \beta \lambda(z)}
$$

then

$$
\begin{gathered}
\frac{d f}{d z}=i \beta e^{i \beta \lambda(z)} \frac{d \lambda}{d z} \\
\frac{d^{2} f}{d z^{2}} \cong-\beta^{2} e^{i \beta \lambda(z)}\left(\frac{d \lambda}{d z}\right)^{2}
\end{gathered}
$$

By substituting these into the homogeneous equation corresponding to Equation [40], one has

$$
\frac{d \lambda_{1,2}}{d z}=\frac{1}{(\gamma+1)(1-z)}\left[1 \pm \sqrt{1+\frac{\gamma+1}{2} \frac{1-z}{z}}\right]
$$

Thus

$\lambda_{1}(z)=\frac{1}{\gamma+1} \int_{z_{1}} \frac{d z}{1-z}\left[1+\sqrt{1+\frac{\gamma+1}{2} \frac{1-z}{z}}\right], \quad \lambda_{1}\left(z_{1}\right)=0$

and

$$
\lambda_{2}(z)=\frac{1}{\gamma+1} \int_{z_{1}} \frac{d z}{1-z}\left[1-\sqrt{1+\frac{\gamma+1}{2} \frac{1-z}{z}}\right]
$$

$$
\lambda_{2}\left(z_{1}\right)=0
$$

The complete solution for large $\beta$ is thus

$$
\begin{array}{r}
f(z) e^{i \omega t=-\frac{\epsilon}{\gamma} e^{i \beta}\left[\frac{u_{2}}{x_{2}} t-\frac{1}{2} \log \left(\frac{z}{z_{1}}\right)\right]}+B e^{i \beta\left[\frac{u_{2}}{x_{2}} t+\lambda_{1}(z)\right]}+ \\
\left.D e^{i \beta\left[\frac{u_{2}}{x_{2}} t+\lambda_{2}(z)\right.}\right] \ldots \ldots
\end{array}
$$

The first term is the particular integral; it satisfies the condition of downstream propagation of disturbances. The same condition requires, however, that $\lambda(z)$ be negative and increasingly negative as $z$ increases from $z_{1}$. $\lambda_{1}(z)$ does not satisfy this condition and hence has to be rejected. Therefore, $B=0 . \quad D$ is fixed by the initial condition of Equation [24], and is $-\epsilon / \gamma(\gamma-1)$. Therefore, finally

$$
f(z)=-\frac{\epsilon}{\gamma}\left[\left(\frac{z}{z_{1}}\right)^{-(i \beta / 2)}+\frac{1}{\gamma-1} e^{i \beta \lambda_{2}(z)}\right] \ldots \ldots[44]
$$

where $\lambda_{2}(z)$ is given Equation [42].

By taking the dominating terms of Equation [22] for large $\beta$, one has

$$
\begin{aligned}
g(z)=f(z)- & (\gamma+1)(1-z) \frac{1}{i \beta} \frac{d f}{d z}+ \\
& \epsilon\left(\frac{z}{z_{1}}\right)^{-(i \beta / 2)}\left[\frac{\gamma+1}{2 \gamma} \frac{1}{z}-\frac{\gamma-1}{2 \gamma}\right]
\end{aligned}
$$

with $f(z)$ as given Equation [44], then $g(z)$ is determined as

$$
g(z)=-\frac{\epsilon}{\gamma(\gamma-1)} \sqrt{1+\frac{\gamma+1}{2} \frac{1-z}{z}} e^{i \beta \lambda_{2}(z),}
$$

$\beta \gg 1$ 
Therefore, the transfer function $G(\beta)$ for large $\beta$ is

$$
G(\beta)=1+\frac{1}{\gamma} \sqrt{1+\frac{\gamma+1}{2} \frac{1-z_{1}}{z_{1}}}, \quad \beta \gg 1
$$

But $z_{1}$ is specified by Equation [23], therefore

$$
G(\beta)=1+\frac{1}{\gamma M_{1}}, \quad \beta \gg 1 \ldots \ldots \ldots \ldots[46]
$$

Therefore, for large frequencies the transfer function is again real and positive, i.e., the mass flow oscillations and the pressure oscillations at the entrance to the nozzle are again in phase. However, the mass flow oscillations are considerably larger than the pressure oscillations; the ratio is given by Equation [46].

\section{Concluding Remarks}

In the previous section the calculation of the transfer function of the rocket nozzle is considered as a problem in nonsteady one-dimensional flow. For very small frequencies of oscillations, the transfer function is indeed approximately equal to unity, as assumed by previous investigators of combustion instability. However, the present calculation also shows that the transfer function is a complex number and has a lead component increasing with increase in frequency. For very large frequencies, the analyses give results indicating larger mass flow oscillations than supposed before. But the transfer function for infinite frequency $\beta$ is again a positive real number. Therefore, the Nyquist diagram of the transfer function $G(\beta)$ is a curve, not a line. As an example, for $\gamma=1.22$, and $M_{1}=0.2$, according to Equations [39] and [46]

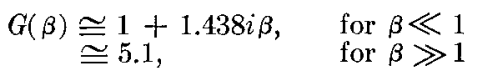

The accuracy of the present results is limited by the underlying assumptions. First of all, the nozzle shape is such as to give a linear velocity distribution in the nozzle. Then the gas is assumed to be perfect and nonreacting. In reality, viscosity, heat conduction, and residual chemical reactions in the exhaust gas will modify the result. At large frequencies or for very short nozzles, the wave length may be small in comparison with the nozzle length, then the one-dimensional flow assumption may introduce appreciable error. For these reasons, it is desirable to measure the transfer function of the rocket nozzle experimentally. Here, however, one must remember that the flow phenomenon in connection with the combustion stability is profoundly influenced by the entropy oscillations generated in the combustion processes. Direct stability measurements on rocket motor with artificially oscillated propellant flow will be necessary. The present analysis indicates, however, that the nozzle could be cut short with practically no divergent part as the transfer function is not influenced by the particular exit velocity so long as the exit velocity is supersonic. The propellant flow rate will then be smaller than a fulllength nozzle, a desirable saving in experimentation. The present investigation also gives the characteristic nondimensional frequency as the angular frequency $\omega$ divided by the velocity gradient along the nozzle. The transfer functions for rocket nozzles of different sizes can be correlated through this nondimensional frequency or reduced frequency.

In conclusion then, it is certain that the present paper gives only a general outline of the problem of transfer function of rocket nozzles. The complete detail has yet to be filled in by further studies.

\section{References}

1 "Stability of Flow in a Rocket Motor," by D. F. Gunder and D. R. Friant, Journal of Applied Mechanics, vol. 17, no. 3, September 1950, p. 327.

2 "A Theory of Unstable Combustion in Liquid Propellant Rocket Systems," by M. Summerfield, Journal of the American Rocket Society, vol. 21, no. 5, September 1951, p. 108.

3 "Aspects of Combustion Stability in Liquid Propellant Rocket Motors, Part I," by L. Crocco, Journal of The AmeriCan Rocket Society, vol. 21, no. 6, November 1951, p. 163.

4 "Aerodynamic Stability and Automatic Control," by W. Bollay, Journal of the Aeronautical Sciences, vol. 18, no. 9, 1951, p. 569 , particularly p. 605 . 